\title{
Somatic driver mutations in endometriosis as possible regulators of fibrogenesis (Review)
}

\author{
HIROSHI KOBAYASHI \\ Department of Obstetrics and Gynecology, Nara Medical University, Kashihara, Nara 634-8522, Japan
}

Received March 31, 2019; Accepted May 3, 2019

DOI: $10.3892 /$ wasj.2019.12

\begin{abstract}
The aim of this review article was to assess the potential link between somatic driver mutations in endometriosis and the pathogenesis of fibrotic processes in other organ systems. This review presents the results of a PubMed literature search for publications dealing with the association between the endometriosis susceptibility driver gene mutations and pathological gene alterations related to fibrosis in the liver, kidney and lung. Genetic studies have demonstrated that endometriosis has somatic mutations in driver genes, including AT-rich interaction domain 1A (ARIDIA), phosphatase and tensin homolog (PTEN), phosphatidylinositol-4,5-bisphosphate 3-kinase catalytic subunit alpha (PIK3CA), KRAS, tumor protein P53 (TP53), P16 or B-Raf proto-oncogene, serine/threonine kinase $(B R A F)$, directly related to neoplasms. Tissue injury and repair induce inflammation and oxidative stress, which induces (epi)genetic DNA damage and mutations, promotes epithelial-mesenchymal transition (EMT) and fibroblast-to-myofibroblast transdifferentiation (FMT) through the induction of fibrogenic signaling pathways regulated by transforming growth factor (TGF)- $\beta / \mathrm{Smad}$ and these driver genes, resulting in $\alpha$-smooth muscle actin $(\alpha$-SMA) and collagen production and increased cellular contractility, leading to increased smooth muscle metaplasia (SMM) and fibrosis. Elevated levels of reactive oxygen species may lead to increased damage to DNA and may induce cell cycle arrest accompanied by the acquisition of replication stress, senescence-associated characteristics and carcinogenesis, at least to a certain extent. This process may be a major hallmark of fibrogenesis of the liver, kidney and lung. Given that somatic driver mutations occur frequently in benign endometriosis and the physiologically normal endometrium, there may be at least 2 distinct phases of epigenetic and genetic modifications in endometriosis: The initial wave of hemoglobin-induced
\end{abstract}

Correspondence to: Professor Hiroshi Kobayashi, Department of Obstetrics and Gynecology, Nara Medical University, 840 Shijo-cho, Kashihara, Nara 634-8522, Japan

E-mail: hirokoba@naramed-u.ac.jp

Key words: endometriosis, myofibroblast, senescence, fibrogenesis, carcinogenesis oxidative stress and (epi)genetic DNA damages and mutations would be followed by the second big wave of cellular senescence, fibrogenesis and finally, carcinogenesis. Thus, it can be concluded that endometriosis may represent a hemorrhageinduced fibrotic and low-grade pre-neoplastic lesion that may be a precursor lesion of a subset of malignant transformation.

\section{Contents}

\section{Introduction}

2. Data collection methods

3. The driver genes in endometriosis as a major hallmark of fibrosis in other organ systems

4. Endometriosis as a fibrotic condition

5. Endometriosis as a premalignant condition

6. Conclusions and future considerations

\section{Introduction}

Among women with endometriosis, approximately $60 \%$ have endometriosis-related pelvic pain, up to $50 \%$ have infertility (1) and approximately $1 \%$ of ovarian endometriomas give rise to ovarian cancer (2). The finding that a large number of somatic driver mutations occur frequently in the physiologically normal epithelial compartment of benign endometriotic lesions without concurrent cancer was surprising $(3,4)$. The repeated hemorrhage in endometriotic lesions contains erythrocytes and their degradation products, such as hemoglobin, heme and free iron. A previous study demonstrated a genetic overlap between the genes differentially expressed in endometriosis and those regulated by the master oxidative stress regulator, iron (5). Alterations in the redox balance or the oxidant/antioxidant pathway can affect the susceptibility to ovarian cancer (5). Oxidative stress, chronic damage and inflammation in endometriosis can lead to epigenetic alterations and genetic mutations of the driver genes, including tumor protein P53 (TP53), phosphatase and tensin homolog $(P T E N)$, AT-rich interaction domain 1A (ARIDIA), phosphatidylinositol-4,5-bisphosphate 3-kinase catalytic subunit alpha (PIK3CA), KRAS and protein phosphatase 2 scaffold subunit Aalpha (PPP2R1A) (6) by altering (epi)genetic signaling in the endometriotic microenvironment (7). Furthermore, Guo previously demonstrated that frequently, mutated driver genes 
in endometriosis may play important roles in fibrogenesis, but do not necessarily contribute to malignant transformation (3). This hypothesis may provide a potential link between somatic driver mutations and the progression of fibrosis. However, to date, at least to the best of our knowledge, there are no studies available reporting whether these genes are required for the development of endometriosis-related fibrosis. At least 3 different types of examples of possibilities on these somatic driver mutations in endometriosis were selected to be shown in this review: i) A characteristic mutational landscape may be an intrinsic molecular processes of normal endometrium; ii) somatic mutations may be required to acquire a self-defined fitness function of the endometriotic lesions under a fluctuating environment during the initiation and progression of endometriosis; and iii) they may not necessarily predict malignancy or pre-malignancy, but the result of selection pressure for fibrogenesis $(3,4,8)$. In this review article, we discuss the mechanisms through which somatic mutations or the abnormal expression of endometriosis-susceptibility driver genes can cause fibrosis in other organ systems.

\section{Data collection methods}

A computerized literature search was conducted to identify relevant studies reported in the English language. We conducted a literature search of the PubMed and Embase databases up to December 2018, combining the keywords 'endometriosis', 'fibrosis', 'fibrogenesis', 'carcinogenesis', 'liver', 'kidney', 'lung', 'mutations', 'genetics' and 'epigenetics'. A variety of combinations of these terms was used, depending on which database was searched. Furthermore, the references of each article were searched to identify potentially relevant studies. The publications of original studies and review articles were included, while those documenting opinions, points of view or anecdotes were discarded.

\section{The driver genes in endometriosis as a major hallmark of fibrosis in other organ systems}

This review presents the results of a literature search for publications dealing with the association between the endometriosis susceptibility driver gene mutations and pathological gene alterations related to fibrosis in the liver, kidney and lung.

Hepatic fibrosis. Hepatic fibrosis is an excessive woundhealing repair following chronic injury to the liver, caused by virus and parasite infections, alcohol abuse, non-alcoholic hepatosteatosis (NASH), metabolic disorders, or autoimmune imbalances (9). Epidemiologically, progressive hepatic fibrosis is a precursor for the development of cirrhosis and hepatocellular carcinoma (9). Activated hepatic stellate cells (HSCs) are the primary source of myofibroblasts (the transdifferentiation of quiescent cells into proliferative, fibrogenic myofibroblasts), the major players of fibrogenesis, that produce extracellular matrix (ECM) proteins (10). The possible molecular mechanisms of hepatic fibrosis are associated with extrinsic and intrinsic factors, including angiogenesis, tissue-degrading enzymes, oxidative stress, inflammatory signaling, autophagy, or cellular senescence. Angiogenesis-related genes are key players in the pathogenesis of hepatic fibrosis and include the profibrotic cytokine, transforming growth factor $\beta$ $(T G F-\beta)$, platelet-derived growth factor $(P D G F)$, connective tissue growth factor $(C T G F)$, hepatocyte growth factor $(H G F)$, tumor necrosis factor- $\alpha(T N F-\alpha)$, basic fibroblast growth factor $(b F G F)$ and vascular endothelial growth factor $(V E G F)(11)$. Changes in the fine balance between tissuedegrading matrix metalloproteinases (MMPs) and their counterparts, tissue inhibitors of metalloproteinases (TIMPs), which drives extracellular matrix turnover, may be critical to maintaining wound healing and ECM homeostasis (12). Tissue remodeling and fibrosis might be controlled by the MMPs/TIMPs imbalance (12). Reactive oxygen species (ROS) can activate HSCs and the progression of fibrosis, resulting in an increased collagen production and ECM deposition (13). ROS are generated through oxidative stress-related lipid peroxidation (13) and inflammatory responses via Toll-like receptors (TLRs) (14). TLR4 and TLR9 signaling pathways play a role in the liver inflammation-fibrosis-carcinoma axis (14).

The roles of driver genes in hepatic fibrosis. The driver genes in hepatic fibrosis are as follows: i) ARIDIA: The mutation of $A R I D I A$, an SWI/SNF chromatin-remodeling gene, induces chromatin remodeling dysfunction and contributes to carcinogenesis (15). The differentially expressed genes (DEGs) and the most commonly mutated genes observed in human hepatocellular carcinoma are TP53, ARIDIA, PTEN, cyclin-dependent kinase inhibitor $2 \mathrm{~A}\left(C D K N 2 A, p 16^{I N K 4 A}\right)$, cyclin D1 (CCND1), catenin beta 1 (CTNNB1), telomerase reverse transcriptase (TERT) and axin 1 (AXIN1) (16). $A R I D 1 A$ deficiency has been shown to be associated with the proliferation, invasion and metastasis of human hepatocellular carcinoma after initiation (15). Aridla expression is downregulated in regenerating tissues, and the loss of Aridla has been shown to increase proliferation and reduce fibrosis following liver injury in an animal model (17). ARIDIA has a negative impact on regeneration after hepatic injury (17). Thus, ARIDIA may be a susceptible gene for hepatic damage and fibrosis. ii) PTEN: PTEN functions as a tumor suppressor by opposing the PI3K/AKT signaling pathway, indicating that the inactivation of PTEN by loss-of-function mutations leads to an aggressive cancer phenotype (18). The PTEN gene is frequently hypermethylated in hepatocellular carcinoma (16). The loss of PTEN plays a role in protection against hyperglycemia and insulin resistance, which results in the development of steatosis, steatohepatitis and NASH (12), as well as in the progression of hepatic fibrosis (19). iii) PIK3CA: The PI3K/Akt signaling pathway is a downstream target of TGF- $\beta$. The TGF- $\beta / \mathrm{PI} 3 \mathrm{~K} / \mathrm{Akt}$ pathway is activated in pulmonary fibrosis through the induction of cell proliferation and ECM synthesis (20). Therefore, the inhibition of this signaling pathway ameliorates hepatic fibrosis (20).iv) KRAS: Activating mutations of $K$-ras or the sustained activation of K-ras expression stimulates HSCs activation, hepatic cholestasis and fibrosis, possibly through the upregulation of $T G F-\beta 2$ and $C T G F$ gene expression (21). v) TP53: In response to genotoxic insults, TP53 serves multiple biological functions, including cellular responses to DNA damage, cell cycle arrest, DNA repair, apoptosis, senescence and fibrosis (22). TP53 promotes HSCs activation and hepatic fibrosis via the upregulation of fibrogenic genes, such as TGF- $\beta$, Wnt/ $\beta$-catenin, $C T G F$, or Sonic hedgehog $(S H H)(22)$ vi) BRAF: The activity of the 
RAF/MEK/ERK signaling pathway is critical for cell cycle arrest, ECM synthesis and hepatic fibrosis (23).

Considering that the majority of driver genes observed in benign endometriosis are required for EMT, FMT, ECM production and hepatic fibrosis, somatic mutations in "cancerassociated genes' are insufficient for malignant transformation.

Renal fibrosis. We then aimed to address whether these driver genes are central elements of EMT, FMT, ECM production, senescence and fibrogenesis in the kidney. Renal tubulointerstitial fibrosis is a common pathophysiological event of chronic kidney disease (24). Chronic kidney disease causes increased inflammation, oxidative stress and proximal tubule cell death in the form of apoptosis or senescence, with accelerated fibrosis and reduced renal function (24). Kidney pericytes are considered to be a possible precursor of myofibroblasts, contributing to ECM production, increased tissue stiffness and fibrosis (24). A number of genes that have been reported to be commonly altered in renal fibrosis are TGF- $\beta$, Notch, hypoxiainducible factor 1 subunit $\alpha(H I F-1 \alpha)$, protein kinase $\mathrm{C}$ $(P K C) / E R K, P T E N, P I 3 K / A k t, K R A S, T P 53$, nuclear factor $\kappa \mathrm{B}$ subunit $1(N F-\kappa B), B R A F, C D K N 2 A$, angiotensin II/ROS and microRNAs (miRNAs or miRs) (25).

The roles of driver genes in renal fibrosis. The driver genes in renal fibrosis are as follows: i) ARIDIA: No data are available for this gene in this context, at least to the best of our knowledge. ii) PTEN: TGF- $\beta$ signaling upregulates the Notch signaling pathway, which in turn promotes TGF- $\beta$ signaling, suggesting that Notch signaling plays a critical role in TGF- $\beta 1$-induced fibrosis (26). PTEN in renal proximal cells is suppressed when TGF- $\beta$ signaling is activated (26). Both the TGF- $\beta$ and Notch pathways are involved in myofibroblast differentiation and in the increased production of $\alpha$-smooth muscle actin $(\alpha-$ SMA) and ECM proteins, which results in the development of renal fibrosis via the inhibition of PTEN expression (27). iii) PIK3CA: The TGF- $\beta / \mathrm{PI} 3 \mathrm{~K}$ pathway plays a critical role in the EMT of renal tubular epithelial cells during renal injury (28). PI3K also promotes angiotensin II-induced renal glomerular and tubular injury, suggesting that PI3K may be a core mediator of renal fibrosis (28). iv) KRAS: K-ras activation has been shown to promote ECM production and stimulate renal fibroblast proliferation in a rat model, suggesting the possible role of K-ras activation in renal fibrosis (29). v) TP53: TP53 upregulation induced by HIF-1 $\alpha$ facilitates ECM production and renal fibrosis during ischemic injury and hypoxia through the accumulation of G2/M cells and the activation of profibrotic TGF- $\beta$, CTGF and plasminogen activator inhibitor-1 (PAI-1)-mediated signaling pathways (30). Researches have highlighted the critical role of the hypoxia-activated, TP53-responsive profibrogenic pathway in the kidney. vi) $B R A F$ : The Raf-dependent profibrotic activity stimulates renal fibrosis through Raf/MEK/ERK signaling, demonstrating that the Raf kinase modulates renal fibrosis (31). vii) $C D K N 2 A, p 16^{I N K 4 a}$ : Renal fibrosis is mediated via the increased expression of senescence-associated markers, such as CDKN2A $\left(p 16^{I N K 4 a}\right), C D K N 1 A\left(p 21^{\text {Cipl/Wafl }}\right), T P 53$, RB transcriptional corepressor $1(R b l)$ and $\beta$-galactosidase in renal tubular cells (32). Similar results have been observed with $T G F-\beta$, cytochrome c oxidase subunit I (COXI) and heat shock protein A5 (HSPA5) in aging renal fibrosis (33).
In summary, the driver genes commonly mutated in endometriosis may cause renal fibrosis.

Pulmonary fibrosis. Damage to the alveolar epithelium or the obliteration of alveolar-capillary structures has been proposed to be involved in TGF- $\beta$-induced myofibroblast differentiation and fibrosis (34). ROS are produced by the mitochondria and NADPH oxidase induces EMT, FMT and fibrosis by secreting TGF- $\beta$ expression, which is the initiating factor in pulmonary fibrosis (35).

The roles of driver genes in pulmonary fibrosis. The driver genes in pulmonary fibrosis are as follows: i) ARIDIA: No data are available for this gene in this context, at least to the best of our knowledge. ii) PTEN: The overexpression of PTEN may inhibit TGF- $\beta 1$-mediated myofibroblast differentiation by attenuating signaling via the TGF- $\beta / \mathrm{Smad} 3$ - and PI3K/Akt-induced production of $\alpha$-SMA, MMP-2 and MMP-9 in the lung (36). Pulmonary fibrosis has been shown to be associated with decreased PTEN expression, increased senescence and the activation of NF- $\mathrm{KB}$ (37). Therefore, the loss of PTEN may induce pulmonary fibrosis via alveolar epithelial cell senescence through the DNA damage-induced NF- $\kappa \mathrm{B}$ activation (37). iii) PIK3CA: The TGF- $\beta / \mathrm{PI} 3 \mathrm{~K} / \mathrm{AKT} / \mathrm{mTOR}$ pathway in pathological myofibroblasts contributes to the induction of pulmonary fibrosis (38). iv) KRAS: The aberrant expression of K-ras protein and mutation in the codon 12 of $K$-ras gene in type II alveolar pneumocytes are associated with the deterioration of pulmonary fibrosis, loss of functions and the induction of lung carcinoma in patients with idiopathic pulmonary fibrosis (IPF) (39). v) TP53: The senescence marker, TP53, drives the fibrotic signaling cascade (40). The senescence of alveolar type 2 cells is implicated in the pathogeneses of pulmonary fibrosis through activating TP53-p21-Rb pathway (41). TP53 (62.9\%) and BRAF (17.1\%) have been found to be significantly mutated in IPF (41). vi) $C D K N 2 A, p 16^{I N K 4 a}$ : Cellular senescence markers, including $p 16^{I N K 4 a}$, induce interstitial remodeling, ECM deposit and pulmonary fibrosis (42). vii) $B R A F: B R A F$ has been shown to be overexpressed and mutated in IPF samples (43). The high incidence of $B R A F$ mutations has been observed in pulmonary fibrosis, most notably the $B R A F^{V 600 E}$ mutation profile (43). viii) NOTCH1: $T G F-\beta / N O T C H 1$ signaling induces myofibroblast differentiation in pulmonary fibrosis (44).

These data provide evidence of a role for the endometriosis susceptibility driver genes in pulmonary fibrosis.

In conclusion, the multifactorial etiology includes repetitive tissue injury and repair, oxidative stress, the overproduction of reactive oxygen species and inflammation-related signaling processes, which promotes myofibroblast differentiation, cellular senescence, excessive ECM synthesis along with organ fibrosis. It is confirmed that the mutated driver pathways or gene sets identified in endometriosis may contribute to tissue fibrosis in the liver, kidney and lung.

\section{Endometriosis as a fibrotic condition}

Under a number of pathological conditions, endometriotic lesions promote EMT and the transdifferentiation of fibroblasts into myofibroblasts (FMT) in response to the TGF- $\beta / \mathrm{Smad}$ signaling pathway, resulting in the formation of exaggerated 
accumulation of $\alpha$-SMA and collagen, leading to increased smooth muscle metaplasia (SMM) and fibrosis (45). Scarring or excess fibrosis may lead to alterations of tissue function, which results in chronic pain and infertility as endometriosis develops. Scarring regains and maintains tissue integrity, although fibrosis induces adverse changes in tissue architecture and the loss of physiological functions. Fibrosis represents consistent features of 3 different types (peritoneal, ovarian, or deep) of endometriosis (46). Older ovarian endometriomas have a higher collagen content and a greater extent of fibrosis than fresh ones (47). The degree of aggregated smooth muscle component and fibrosis, so-called SMM, is not an uncommon phenomenon in endometriosis (48). Among peritoneal, ovarian and rectovaginal (deep infiltrating) endometriotic lesions, SMM is the most common finding in deep infiltrating endometriosis (48).

Several elegant reviews have been published focusing on the link between endometriosis and fibrosis thus far. The fibrosis-related factors include $T G F-\beta$, neuropeptides substance $\mathrm{P}(S P)$, neurokinin receptor $1(N K 1 R)$, calcitonin receptor like receptor $(C R L R)$, calcitonin gene related peptide $(C G R P)$, receptor activity modifying protein $1(R A M P-1)$, nuclear receptor subfamily 4 group A member 1 (NR4A1), nuclear factor, erythroid 2 like 2 (NFE2L2, also known as $N r f 2)$, or glutamate cysteine ligase $(49,50)$. Among these fibrosis susceptibility genes, TGF- $\beta$ is considered to be a key mediator in a number of fibrotic disorders, including endometriosis, and in fibrosis in hepatic, renal, pulmonary and cardiovascular systems (1). Activated TGF- $\beta$ is released by platelets, macrophages, or myofibroblasts and positively regulates mitochondrial NADPH oxidase 4 (NOX4), which augments ROS generation (34). ROS signaling also leads to the activation of TGF- $\beta$ (51). Therefore, ROS and NOX4 form a positive feedback loop to amplify TGF- $\beta$ signaling, which causes the deterioration of endometriosis and promotes fibrogenesis. Furthermore, platelets activated by excessive hemorrhaging drive SMM and fibrogenesis in endometriosis through EMT and FMT (52). SP, a neuropeptide expressed in peripheral sensory neurons, can stimulate the development EMT, FMT, SMM and ultimately, fibrosis, in endometriotic lesions, indicating that the main clinical features are chronic pelvic pain (53).

It has been proposed that when normal repair mechanisms breakdown, the injured tissues initiate proliferation, myofibrogenesis or various diseases, including cancers, at least to a certain extent. Cellular senescence may be one of the major mechanisms involved in the beneficial and deleterious effects of tissue injury-induced fibrogenesis. Tissue injury has been proposed to promote a number of DNA damage response mechanisms and to repair signaling cascades that control cell cycle arrest to allow DNA damage repair and induce cellular senescence and cell fate (54). Cellular senescence promotes the renewal of damaged tissues. Damage accumulation stimulates the activity of CDK inhibitors, CDKN2A ( $\left.p 16^{\operatorname{lnk} 4 a}\right)$, CDKN1A ( $p 21^{\text {Cipl/Wafl })}$, CDKN2B $\left(p 15^{\text {Ink4b }}\right)$, or TP53, which antagonizes CDKs to block cell cycle progression and induce senescence (54). Thus, the CDKN families are essential for the maintenance of the senescent-cell-cycle arrest. Not only CDK inhibitors, but also the transcription factor, hepatocyte nuclear factor $(H N F)-1 \beta$, are significantly upregulated in endometriosis and its malignant transformation, clear cell carcinoma of the ovary (55). HNF-1 $\beta$ is considered to promote G2/M cell cycle arrest in response to DNA damage through the HNF-1 $1 /$ ubiquitin specific peptidase 28 (USP28)/Claspin/Chk1 signaling pathway (56). Cellular senescence is recognized as the state of irreversible cell cycle arrest in response to a variety of various intrinsic and extrinsic cellular stresses (57) and sometimes evolves as a beneficial response to protection against tissue fibrosis (58). On the other hand, cellular senescence also entails irreversible replicative arrest and acquires senescenceassociated secretory phenotype that causes endometriotic cells to become susceptible to their own harmful microenvironment and gradually accumulates DNA damage, and even the promotion of tumorigenesis (59). Hypotheses involving cellular senescence will be developed, from two points of view: Senescence appears to be beneficial or deleterious.

An interesting study demonstrated that numerous somatic mutations were identified, not only in benign endometriosis, but also in the normal endometrium (8). Suda et al discussed the impact of intrinsic molecular processes on mutation acquisition in the normal endometrium (8). Repeated hemorrhaging during menstruation and in endometriotic lesions leads to severe hemolysis that results in high levels of hemoglobin, heme and free iron. Autoxidation and fenton reaction of hemoglobin from the ferrous $\mathrm{Fe}^{2+}$ (oxyhemoglobin) state to the ferric $\mathrm{Fe}^{3+}$ (methemoglobin) state lead to the production of excess ROS, such as $\mathrm{O}_{2-}$ and $\cdot \mathrm{OH}$, which mediate oxidative stress and promote DNA damage and mutations (60). DNA mutations provide benefits that are essential for the survival of endometriotic cells and normal endometrial cells exposed regularly to menstrual blood. These cells may be adapted or selected to survive under the conditions of oxidative stress.

Recent data have indicated a fundamental role of oxidative stress, secondary to the influx of hemoglobin, heme and free iron, in expressing $\mathrm{CpG}$ demethylases, ten-eleven translocation (TET) and jumonji (JMJ) genes (61). Both TET and $J M J$ genes recognize a wide range of endogenous DNA methyltransferases (DNMTs) (61). The expression levels of DNMTs may be involved in the subsequent epigenetic modulation of endometriosis susceptibility genes (61). Indeed, 8-OHdG immunohistochemical staining, a marker of oxidative DNA damage, has been found in normal ovarian cortex surrounding ovarian endometriomas (62). Elevated levels of ROS may lead to the increased damage of DNA and induce cell cycle arrest accompanied by the acquisition of replication stress, senescence-associated characteristics and carcinogenesis (63). This review supports the hypothesis that there are at least 2 distinct phases of epigenetic and genetic modifications in endometriosis: The initial wave of hemoglobin-induced oxidative stress and (epi)genetic DNA damage and mutations would be followed by the second big wave of cell senescence, fibrogenesis and finally, carcinogenesis.

\section{Endometriosis as a premalignant condition}

The epidemiological, histological, genetic and molecular alterations in endometriosis may suggest that endometriosis is a premalignant condition $(64,65)$. First, there is evidence of a broad overlap between gene mutation clusters for benign endometriosis and its malignant transformation. The candidate 


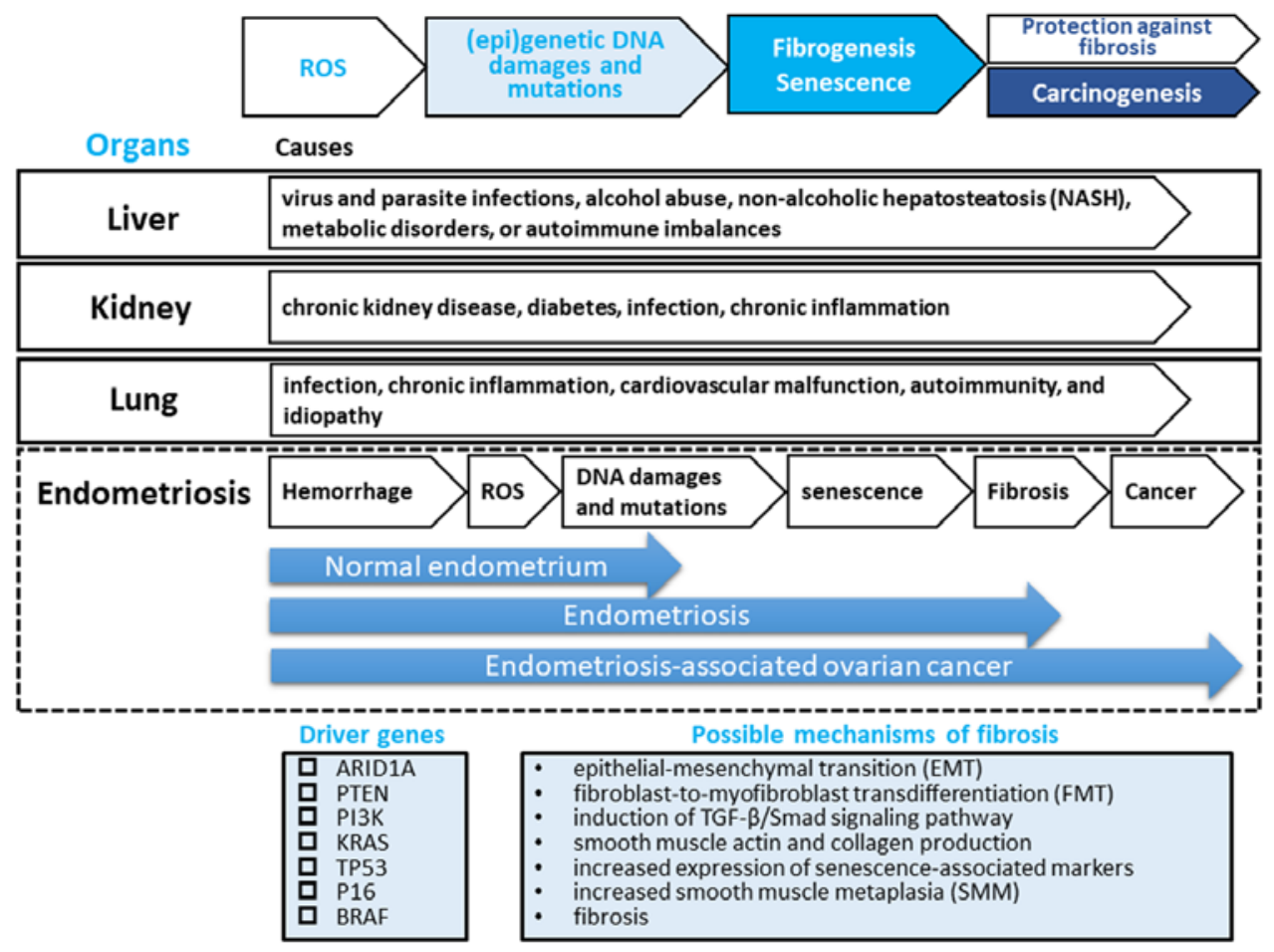

Figure 1. A hypothesis for the pathogenesis of endometriosis fibrogenesis. Genetic studies have demonstrated that endometriotic lesions have somatic mutations in driver genes, including AT-rich interaction domain 1A (ARID1A), phosphatase and tensin homolog (PTEN), phosphoinositide 3-kinase (PI3K), $K R A S$, tumor protein P53 (TP53), P16, or B-Raf proto-oncogene, serine/threonine kinase (BRAF), directly related to neoplasms. Tissue injury and repair induce inflammation and oxidative stress, which induces (epi)genetic DNA damage and mutations, promotes epithelial-mesenchymal transition (EMT) and fibroblast-to-myofibroblast transdifferentiation (FMT) through the induction of the transforming growth factor (TGF)- $\beta /$ Smad signaling pathway, resulting in $\alpha$-smooth muscle actin ( $\alpha$-SMA) and collagen production and increased cellular contractility, leading to increased smooth muscle metaplasia (SMM) and fibrosis (45). Elevated levels of reactive oxygen species (ROS) may lead to increased damage to DNA and induce cell cycle arrest accompanied by the acquisition of replication stress, senescence-associated characteristics and carcinogenesis, at least to a certain extent (63). This process may be a major hallmark of fibrogenesis of liver, kidney and lung. There may be at least 2 distinct phases of epigenetic and genetic modifications in endometriosis: the initial wave of hemoglobin-induced oxidative stress and (epi)genetic DNA damage and mutations would be followed by the second big wave of cell senescence, fibrogenesis and finally carcinogenesis. Therefore, endometriosis may represent a hemorrhage-induced pre-fibrotic and low-grade neoplastic lesion that may be a precursor lesion of a subset of malignant transformation of endometriosis. Our hypothesis is not inconsistent with available evidence that a type I tumor exhibits an adaptive stepwise progression from normal endometrium, through endometriosis and fibrosis, to endometriosis-associated ovarian cancer.

mutated driver gene sets, including ARIDIA, PIK3CA, $P T E N, T P 53, K R A S$ and PPP2RIA, have been identified in endometriosis $(6,7)$. More recently, Lac et al reported that hotspot mutations in $K R A S$, erb-b2 receptor tyrosine kinase 2 (ERBB2), PIK3CA and CTNNB1, heterogeneous PTEN loss and ARIDIA loss were also identified in endometriosis (66). Genetic studies have demonstrated that endometriotic lesions have somatic mutations in genes directly related to endometriosis-associated ovarian cancer (6,7,64-66). It has been established that gene mutations are found in benign endometriotic lesions adjacent to invasive carcinomas having mutations (67). However, the same mutations have also been described in benign endometriosis that do not coincide with synchronous carcinoma specimens or that never progress to malignancy (66). Studies have shown a significant overlap in driver mutations between endometriosis with or without coexistent malignancy $(6,7,66,67)$.

Second, a recent study by Suda et al reported that numerous somatic mutations were identified in both endometriotic and normal epithelium samples (8). The eutopic endometrium may share similarities with the ectopic endometrium at the environmental level: Erythrocytes release some inflammatory mediators, including oxidative stress and ROS, into the uterine cavity, peritoneal cavity, or into cysts.
A previous study demonstrated a global overlap in the genes differentially expressed in endometriosis and those modulated by the oxidative stress regulator, iron (5). Alterations in the redox balance can explain a part of the somatic oncogene mutations (5). For example, oxidative stress, in the form of lipid peroxidation and 4-hydroxy-2-nonenal, has been linked to site-specific mutations of the TP53 gene (68). A positive association has also been shown between heme iron intake and the risk of colorectal carcinoma with activating $\mathrm{G}>\mathrm{A}$ mutations in $K R A S(69)$. The iron-induced genetic mutations that may arise from the formation of $8-\mathrm{OHdG}$ involve $\mathrm{GC} \rightarrow \mathrm{TA}$ transversions (70). Epidemiological, biological and molecular data suggest that endometriosis is a risk factor for the later development of several types of cancer, including endometrial cancer and ovarian cancer $(64,65)$. The key genetic alterations occur as a normal endometrium and progress to endometriosis, and ultimately tumor cells, which may explain the reasons and mechanisms though which the normal endometrium can change into endometriosis and subsequently into several types of cancer. However, the exact molecular mechanisms underlying the conversion of the normal endometrium to endometriosis and then cancers remain uncertain.

Finally, it is of interest that cancer, endometriosis and the normal endometrium often share common pathogenetic 
determinants, such as DNA damage, oxidative stress, chronic inflammation and other chronic conditions, such as the longer duration of menstruation and hemorrhaging. This article outlined the possibility of endometriosis fibrogenesis, possibly through driver genes controlling malignant transformation. This review supports novel conceptual ideas that frequently mutated driver genes in endometriosis may initially play dominant roles in the initiation and progression of fibrogenesis, although these genetic abnormalities alone are insufficient for malignant transformation.

\section{Conclusions and future considerations}

Given that somatic driver mutations occur frequently in the physiologically normal endometrium and benign endometriosis, herein, we reviewed the functions of these driver genes (ARIDIA, PTEN, PI3K, KRAS, TP53, CDKN2A and BRAF) in EMT, FMT, ECM production and increased SMM during fibrosis in liver, kidney and lung (6,7,64-66). To date, much has been shown about a complex pathway model for fibrogenesis based on common driver genes. The driver molecules related to the progression of fibrosis are $T G F-\beta$, Notch, HIF-1 $\alpha$, PTEN, PIK3CA, PI3K/Akt, KRAS, TP53, NF- $\kappa B, B R A F$, $C D K N 2 A$ and $A R I D 1 A$ that can activate myofibrogenesis, resulting in increased ECM production, collagen deposition and fibrosis (11-13,16,22,25,32,49,50,54). A significant overlap was observed between 2 sets, epigenetic alterations and genetic mutations of the driver genes in endometriosis and the driver molecules related to the progression of fibrosis in the liver, kidney and lung. This review provides support for the hypothesis that these driver genes can accelerate the process of fibrogenesis in endometriosis (Fig. 1).

In both endometriotic and normal epithelium samples, numerous somatic mutations have been identified within genes frequently mutated in endometriosis-associated ovarian cancers (8). Perhaps the greatest uncertainty is not why endometriosis spontaneously develops into fibrosis, but rather why the normal endometrium with similar driver mutations does not. Endometriosis, but not the normal endometrium, are prone to repetitive hemorrhage-induced tissue injury and repair, which persistently induces oxidative stress, the overproduction of ROS and inflammation-related signaling. We made the following hypothesis that long-term damage to the endometriotic lesions leads to excess oxidative stress, inflammation, senescence, myofibroblast differentiation and ultimately fibrosis, whereas excessive menstruation may cause a series of repetitive injuries to the eutopic endometrial tissue resulting in an altered healing process, which changes the architecture of the uterine myometrium, leading to the development of adenomyosis.

In conclusion, the aberrant expression or somatic genetic mutations in TP53, PTEN, ARIDIA, PIK3CA and KRAS identified in endometriosis may not be limited to early stages of cancer evolution, but cause fibrosis in various organs. Future studies are warranted to summarize the mechanisms and functions of the somatic driver landscape of endometriosis affecting fibrogenesis.

\section{Acknowledgements}

Not applicable.

\section{Funding}

No funding was received.

\section{Availability of data and materials}

Not applicable.

\section{Author's contribution}

HK performed the literature search, and collected the data using the PubMed and Embase databases. HK conceived this review article. HK designed this review article and interpreted the included research studies. The final version of the manuscript has been read and approved by HK.

\section{Ethics approval and consent to participate}

Not applicable.

\section{Patient consent for publication}

Not applicable.

\section{Competing interests}

The author declares that there are no competing interests.

\section{References}

1. Giudice LC: Clinical practice. Endometriosis. N Engl J Med 362: 2389-2398, 2010

2. Anglesio MS and Yong PJ: Endometriosis-associated Ovarian Cancers. Clin Obstet Gynecol 60: 711-727, 2017.

3. Guo SW: Cancer driver mutations in endometriosis: Variations on the major theme of fibrogenesis. Reprod Med Biol 17: 369-397, 2018.

4. Anglesio MS, Papadopoulos N, Ayhan A, Nazeran TM, Noë M, Horlings HM, Lum A, Jones S, Senz J, Seckin T, et al: Cancerassociated mutations in endometriosis without cancer. $\mathrm{N}$ Engl J Med 376: 1835-1848, 2017.

5. Kobayashi H, Yamada Y, Kanayama S, Furukawa N, Noguchi T, Haruta S, Yoshida S, Sakata M, Sado T and Oi H: The role of iron in the pathogenesis of endometriosis. Gynecol Endocrinol 25: 39-52, 2009.

6. Kurman RJ and Shih IeM: Molecular pathogenesis and extraovarian origin of epithelial ovarian cancer - shifting the paradigm. Hum Pathol 42: 918-931, 2011.

7. Xie H, Chen P, Huang HW, Liu LP and Zhao F: Reactive oxygen species downregulate ARID1A expression via its promoter methylation during the pathogenesis of endometriosis. Eur Rev Med Pharmacol Sci 21: 4509-4515, 2017.

8. Suda K, Nakaoka H, Yoshihara K, Ishiguro T, Tamura R, Mori Y, Yamawaki K, Adachi S, Takahashi T, Kase H, et al: Clonal expansion and diversification of cancer-associated mutations in endometriosis and normal endometrium. Cell Rep 24: 1777-1789, 2018.

9. Iwaisako K, Brenner DA and Kisseleva T: What's new in liver fibrosis? The origin of myofibroblasts in liver fibrosis. J Gastroenterol Hepatol 27 (Suppl 2): 65-68, 2012.

10. Seki E and Brenner DA: Recent advancement of molecular mechanisms of liver fibrosis. J Hepatobiliary Pancreat Sci 22: 512-518, 2015.

11. Wight TN and Potter-Perigo S: The extracellular matrix: An active or passive player in fibrosis? Am J Physiol Gastrointest Liver Physiol 301: G950-G955, 2011.

12. Guo J and Friedman SL: Hepatic fibrogenesis. Semin Liver Dis 27: 413-426, 2007. 
13. Ghatak S, Biswas A, Dhali GK, Chowdhury A, Boyer JL and Santra A: Oxidative stress and hepatic stellate cell activation are key events in arsenic induced liver fibrosis in mice. Toxicol Appl Pharmacol 251: 59-69, 2011.

14. Song IJ, Yang YM, Inokuchi-Shimizu S, Roh YS, Yang L and Seki E: The contribution of toll-like receptor signaling to the development of liver fibrosis and cancer in hepatocyte-specific TAK1-deleted mice. Int J Cancer 142: 81-91, 2018.

15. Abe H, Hayashi A, Kunita A, Sakamoto Y, Hasegawa K, Shibahara J, Kokudo N and Fukayama M: Altered expression of AT-rich interactive domain 1A in hepatocellular carcinoma. Int J Clin Exp Pathol 8: 2763-2770, 2015.

16. Khemlina G, Ikeda S and Kurzrock R: The biology of hepatocellular carcinoma: Implications for genomic and immune therapies. Mol Cancer 16: 149, 2017.

17. Sun X, Chuang JC, Kanchwala M, Wu L, Celen C, Li L, Liang H, Zhang S, Maples T, Nguyen LH, et al: Suppression of the SWI/SNF component Aridla promotes mammalian regeneration. Cell Stem Cell 18: 456-466, 2016.

18. Guigon CJ, Zhao L, Willingham MC and Cheng SY: PTEN deficiency accelerates tumour progression in a mouse model of thyroid cancer. Oncogene 28: 509-517, 2009.

19. Yu F, Chen B, Dong P and Zheng J: HOTAIR epigenetically modulates PTEN expression via microRNA-29b: A novel mechanism in regulation of liver fibrosis. Mol Ther 25: 205-217, 2017.

20. Son MK, Ryu YL, Jung KH, Lee H, Lee HS, Yan HH, Park HJ, Ryu JK, Suh JK, Hong S, et al: HS-173, a novel PI3K inhibitor, attenuates the activation of hepatic stellate cells in liver fibrosis. Sci Rep 3: 3470, 2013

21. Makino Y, Hikita H, Kodama T, Shigekawa M, Yamada R, Sakamori R, Eguchi H, Morii E, Yokoi H, Mukoyama M, et al CTGF mediates tumor-stroma interactions between hepatoma cells and hepatic stellate cells to accelerate HCC progression. Cancer Res 78: 4902-4914, 2018.

22. Kodama T, Takehara T, Hikita H, Shimizu S, Shigekawa M, Tsunematsu H, Li W, Miyagi T, Hosui A, Tatsumi T, et al: Increases in $\mathrm{p} 53$ expression induce CTGF synthesis by mouse and human hepatocytes and result in liver fibrosis in mice. J Clin Invest 121: 3343-3356, 2011.

23. Guo X, Cen Y, Wang J and Jiang H: CXCL10-induced IL-9 promotes liver fibrosis via Raf/MEK/ERK signaling pathway. Biomed Pharmacother 105: 282-289, 2018.

24. Portilla D: Apoptosis, fibrosis and senescence. Nephron Clin Pract 127: 65-69, 2014.

25. Liu M, Ning X, Li R, Yang Z, Yang X, Sun S and Qian Q: Signalling pathways involved in hypoxia-induced renal fibrosis J Cell Mol Med 21: 1248-1259, 2017.

26. Lan R, Geng H, Polichnowski AJ, Singha PK, Saikumar P, McEwen DG, Griffin KA, Koesters R, Weinberg JM, Bidani AK, et al: PTEN loss defines a TGF- $\beta$-induced tubule phenotype of failed differentiation and JNK signaling during renal fibrosis. Am J Physiol Renal Physiol 302: F1210-F1223, 2012.

27. Bielesz B, Sirin Y, Si H, Niranjan T, Gruenwald A, Ahn S Kato H, Pullman J, Gessler M, Haase VH, et al: Epithelial Notch signaling regulates interstitial fibrosis development in the kidneys of mice and humans. J Clin Invest 120: 4040-4054, 2010

28. Zhou T, Luo M, Cai W, Zhou S, Feng D, Xu C and Wang H: Runt-related transcription factor 1 (RUNX1) promotes TGF- $\beta$-induced renal tubular epithelial-to-mesenchymal transition (EMT) and renal fibrosis through the PI3K subunit p1108. EBioMedicine 31: 217-225, 2018

29. Rodríguez-Peña AB, Santos E, Arévalo M and López-Novoa JM Activation of small GTPase Ras and renal fibrosis. J Nephrol 18 341-349, 2005.

30. Liu L, Zhang P, Bai M, He L, Zhang L, Liu T, Yang Z, Duan M, Liu M, Liu B, et al: p53 upregulated by HIF-1 $\alpha$ promotes hypoxiainduced $\mathrm{G} 2 / \mathrm{M}$ arrest and renal fibrosis in vitro and in vivo. $\mathrm{J} \mathrm{Mol}$ Cell Biol: Jul 18, 2018 (Epub ahead of print).

31. Buchholz B, Klanke B, Schley G, Bollag G, Tsai J, Kroening S, Yoshihara D, Wallace DP, Kraenzlin B, Gretz N, et al: The Raf kinase inhibitor PLX5568 slows cyst proliferation in rat polycystic kidney disease but promotes renal and hepatic fibrosis Nephrol Dial Transplant 26: 3458-3465, 2011.

32. Adijiang A, Shimizu H, Higuchi $Y$, Nishijima $F$ and Niwa $T$ : Indoxyl sulfate reduces klotho expression and promotes senescence in the kidneys of hypertensive rats. J Ren Nutr 21: $105-109,2011$.
33. Melk A, Schmidt BM, Takeuchi O, Sawitzki B, Rayner DC and Halloran PF: Expression of p16INK4a and other cell cycle regulator and senescence associated genes in aging human kidney. Kidney Int 65: 510-520, 2004.

34. Jain M, Rivera S, Monclus EA, Synenki L, Zirk A, Eisenbart J, Feghali-Bostwick C, Mutlu GM, Budinger GR and Chandel NS: Mitochondrial reactive oxygen species regulate transforming grow th factor- $\beta$ signaling. J Biol Chem 288: 770-777, 2013.

35. Leask A and Abraham DJ: TGF-beta signaling and the fibrotic response. FASEB J 18: 816-827, 2004.

36. Xie B, Zheng G, Li H, Yao X, Hong R, Li R, Yue W and Chen Y: Effects of the tumor suppressor PTEN on the pathogenesis of idiopathic pulmonary fibrosis in Chinese patients. Mol Med Rep 13: 2715-2723, 2016.

37. Tian Y, Li H, Qiu T, Dai J, Zhang Y, Chen J and Cai H: Loss of PTEN induces lung fibrosis via alveolar epithelial cell senescence depending on NF-KB activation. Aging Cell 18: e12858, 2019.

38. Hsu HS, Liu CC, Lin JH, Hsu TW, Hsu JW, Su K and Hung SC: Involvement of ER stress, PI3K/AKT activation, and lung fibroblast proliferation in bleomycin-induced pulmonary fibrosis. Sci Rep 7: 14272, 2017.

39. Takahashi T, Munakata M, Ohtsuka Y, Nisihara H, Nasuhara Y, Kamachi-Satoh A, Dosaka-Akita H, Homma Y and Kawakami Y: Expression and alteration of ras and p53 proteins in patients with lung carcinoma accompanied by idiopathic pulmonary fibrosis Cancer 95: 624-633, 2002.

40. Álvarez D, Cárdenes N, Sellarés J, Bueno M, Corey C, Hanumanthu VS, Peng Y, D'Cunha H, Sembrat J, Nouraie M, et al: IPF lung fibroblasts have a senescent phenotype. Am J Physiol Lung Cell Mol Physiol 313: L1164-L1173, 2017.

41. Higgins SP, Tang Y, Higgins CE, Mian B, Zhang W, Czekay RP, Samarakoon R, Conti DJ and Higgins PJ: TGF- $\beta 1 / \mathrm{p} 53$ signaling in renal fibrogenesis. Cell Signal 43: 1-10, 2018

42. Kuwano K, Kunitake R, Kawasaki M, Nomoto Y, Hagimoto N, Nakanishi Y and Hara N: P21Waf1/Cip1/Sdil and p53 expression in association with DNA strand breaks in idiopathic pulmonary fibrosis. Am J Respir Crit Care Med 154: 477-483, 1996.

43. Hwang JA, Kim D, Chun SM, Bae S, Song JS, Kim MY, Koo HJ, Song JW, Kim WS, Lee JC, et al: Genomic profiles of lung cancer associated with idiopathic pulmonary fibrosis. J Pathol 244: $25-35,2018$

44. Hu B, Wu Z, Bai D, Liu T, Ullenbruch MR and Phan SH: Mesenchymal deficiency of Notch1 attenuates bleomycininduced pulmonary fibrosis. Am J Pathol 185: 3066-3075, 2015.

45. Zhang Q, Duan J, Olson M, Fazleabas A and Guo SW: Cellular changes consistent with epithelial-mesenchymal transition and fibroblast-to-myofibroblast transdifferentiation in the progression of experimental endometriosis in baboons. Reprod Sci 23: 1409-1421, 2016.

46. Vigano P, Candiani M, Monno A, Giacomini E, Vercellini P and Somigliana E: Time to redefine endometriosis including its pro-fibrotic nature. Hum Reprod 33: 347-352, 2018.

47. Guo SW, Ding D, Shen M and Liu X: Dating endometriotic ovarian cysts based on the content of cyst fluid and its potential clinical implications. Reprod Sci 22: 873-883, 2015.

48. Kim HS, Yoon G, Ha SY and Song SY: Nodular smooth muscle metaplasia in multiple peritoneal endometriosis. Int J Clin Exp Pathol 8: 3370-3373, 2015

49. Yan D, Liu X and Guo SW: Neuropeptides substance P and calcitonin gene related peptide accelerate the development and fibrogenesis of endometriosis. Sci Rep 9: 2698, 2019.

50. Marcellin L, Santulli P, Chouzenoux S, Cerles O, Nicco C, Dousset B, Pallardy M, Kerdine-Römer S, Just PA, Chapron C, et al: Alteration of $\mathrm{Nrf} 2$ and Glutamate Cysteine Ligase expression contribute to lesions growth and fibrogenesis in ectopic endometriosis. Free Radic Biol Med 110: 1-10, 2017.

51. Gonzalez-Gonzalez FJ, Chandel NS, Jain M and Budinger GRS: Reactive oxygen species as signaling molecules in the development of lung fibrosis. Transl Res 190: 61-68, 2017.

52. Zhang Q, Liu X and Guo SW: Progressive development of endometriosis and its hindrance by anti-platelet treatment in mice with induced endometriosis. Reprod Biomed Online 34: 124-136, 2017.

53. Liu X, Yan D and Guo SW: Sensory nerve-derived neuropeptides accelerate the development and fibrogenesis of endometriosis. Hum Reprod 34: 452-468, 2019.

54. van Deursen JM: The role of senescent cells in ageing. Nature 509: 439-446, 2014

55. Kato N, Sasou S and Motoyama T: Expression of hepatocyte nuclear factor-1beta (HNF-1beta) in clear cell tumors and endometriosis of the ovary. Mod Pathol 19: 83-89, 2006. 
56. Ito F, Yoshimoto C, Yamada Y, Sudo T and Kobayashi H: The HNF-1 $\beta$-USP28-Claspin pathway upregulates DNA damageinduced Chk1 activation in ovarian clear cell carcinoma. Oncotarget 9: 17512-17522, 2018.

57. Kadota T, Fujita Y, Yoshioka Y, Araya J, Kuwano K and Ochiya T: Emerging role of extracellular vesicles as a senescence-associated secretory phenotype: Insights into the pathophysiology of lung diseases. Mol Aspects Med 60: 92-103, 2018.

58. Regulski MJ: Cellular senescence: What, why, and how. Wounds 29: 168-174, 2017.

59. Watanabe S, Kawamoto S, Ohtani N and Hara E: Impact of senescence-associated secretory phenotype and its potential as a therapeutic target for senescence-associated diseases. Cancer Sci 108: 563-569, 2017

60. Iwabuchi T, Yoshimoto C, Shigetomi H and Kobayashi H: Oxidative stress and antioxidant defense in endometriosis and its malignant transformation. Oxid Med Cell Longev 2015: 848595, 2015.

61. Ito F, Yamada Y, Shigemitsu A, Akinishi M, Kaniwa H, Miyake R, Yamanaka S and Kobayashi H: Role of oxidative stress in epigenetic modification in endometriosis. Reprod Sci 24: 1493-1502, 2017.

62. Di Emidio G, D'Alfonso A, Leocata P, Parisse V, Di Fonso A, Artini PG, Patacchiola F, Tatone C and Carta G: Increased levels of oxidative and carbonyl stress markers in normal ovarian cortex surrounding endometriotic cysts. Gynecol Endocrinol 30: 808-812, 2014.

63. Baker DJ, Alimirah F, van Deursen JM, Campisi J and Hildesheim J: Oncogenic senescence: A multi-functional perspective. Oncotarget 8: 27661-27672, 2017.
64. Painter JN, O'Mara TA, Morris AP, Cheng THT, Gorman M, Martin L, Hodson S, Jones A, Martin NG, Gordon S, et al: Genetic overlap between endometriosis and endometrial cancer: Evidence from cross-disease genetic correlation and GWAS meta-analyses. Cancer Med 7: 1978-1987, 2018.

65. Ramalingam P: Morphologic, immunophenotypic, and molecular features of epithelial ovarian cancer. Oncology (Williston Park) 30: 166-176, 2016.

66. Lac V, Verhoef L, Aguirre-Hernandez R, Nazeran TM, TessierCloutier B, Praetorius T, Orr NL, Noga H, Lum A, Khattra J, et al: Iatrogenic endometriosis harbors somatic cancer-driver mutations. Hum Reprod 34: 69-78, 2019.

67. Siufi Neto J, Kho RM, Siufi DF, Baracat EC, Anderson KS and Abrão MS: Cellular, histologic, and molecular changes associated with endometriosis and ovarian cancer. J Minim Invasive Gynecol 21: 55-63, 2014.

68. Petersen DR: Alcohol, iron-associated oxidative stress, and cancer. Alcohol 35: 243-249, 2005.

69. Gilsing AM, Fransen F, de Kok TM, Goldbohm AR, Schouten LJ, de Bruïne AP, van Engeland M, van den Brandt PA, de Goeij AF and Weijenberg MP: Dietary heme iron and the risk of colorectal cancer with specific mutations in KRAS and APC. Carcinogenesis 34: 2757-2766, 2013.

70. Valko M, Izakovic M, Mazur M, Rhodes CJ and Telser J: Role of oxygen radicals in DNA damage and cancer incidence. Mol Cell Biochem 266: 37-56, 2004

(i) (9) This work is licensed under a Creative Commons Con Attribution-NonCommercial-NoDerivatives 4.0 International (CC BY-NC-ND 4.0) License. 